\title{
Effects of Mindfulness-Based Cognitive Therapy on a Behavioural Measure of Rumination in Patients with Chronic, Treatment-Resistant Depression
}

\author{
Mira B. Cladder-Micus ${ }^{1,2,3}$ (D) Eni S. Becker ${ }^{1}$ - Jan Spijker ${ }^{1,2} \cdot$ Anne E. M. Speckens ${ }^{3}$ (D) Janna N. Vrijsen ${ }^{2,3}$ (D)
}

Published online: 13 February 2019

(c) The Author(s) 2019

\begin{abstract}
It has been found that Mindfulness-based cognitive therapy (MBCT) reduces rumination in remitted and currently depressed patients. However, less is known about the effects of MBCT on rumination in chronically and treatment-resistant depressed patients. Typically, questionnaires are used to assess rumination, but this introduces the risk of response and recall biases. A recent systematic review (van der Velden et al. Clinical Psychology Review 37:26-39, 2015) proposes to also include behavioural measures. A behavioural measure that has previously been used to assess rumination in dysphoric students is the breathing focus task (BFT). The first aim of this research was to investigate whether the BFT can be used in chronically, treatment-resistant depressed patients to measure rumination. We therefore administered the BFT in patients with chronic, treatment-resistant depression $(n=73)$ and compared them with never-depressed controls $(n=106)$. Patients reported significantly more negative thought intrusions and subsequent sad mood. Secondly we tested in a randomized-controlled trial whether MBCT in combination with treatment-as-usual (MBCT + TAU, $n=26)$ compared with TAU $(n=36)$ reduces rumination assessed with the BFT in chronically, treatment-resistant depressed patients. Negative thought intrusions significantly decreased in the MBCT + TAU condition, compared with TAU. The results show that MBCT reduces rumination assessed with the BFT in chronically, treatment-resistant depressed patients.
\end{abstract}

Keywords Mindfulness $\cdot$ MBCT $\cdot$ Rumination $\cdot$ Depression $\cdot$ RCT $\cdot$ Chronic depression

\section{Introduction}

Mindfulness-based cognitive therapy (MBCT) is an 8-week group training for depression that combines mindfulness meditation techniques with elements of cognitive behavioural therapy (Segal et al. 2012). During MBCT participants are taught to react to thoughts, emotions and bodily sensations in a non-judgmental and compassionate way. In

Mira B. Cladder-Micus

m.cladder-micus@psych.ru.nl

1 Behavioural Science Institute, Radboud University, 6500 HE Nijmegen, The Netherlands

2 Depression Expertise Centre, Pro Persona Mental Health Care, Reinier Postlaan 6, 6525 GC Nijmegen, The Netherlands

3 Department of Psychiatry, Donders Institute for Brain, Cognition and Behaviour, Radboud University Medical Center, Internal Postal Code 966, 6500 HB Nijmegen, The Netherlands the past years, several studies have shown that MBCT effectively reduces relapse rates in remitted depressed patients (Kuyken et al. 2016). Furthermore, research has shown that MBCT significantly decreases depressive symptoms in patients with current major depression (Strauss et al. 2014) and recent studies tested its applicability to more complex forms of depression, such as chronic and treatment-resistant depression (Eisendrath et al. 2016; Cladder-Micus et al. 2018; Michalak et al. 2016).

It is assumed that MBCT decreases depressive symptoms by reducing dysfunctional cognitive processes, specifically depressive rumination (Segal et al. 2012). Nolen-Hoeksema et al. (2008) define depressive rumination as the repetitive dwelling on negative emotions and their causes and implications. During the last decade, researchers (Ehring and Watkins 2008; Harvey et al. 2004) have argued that rumination together with other negative dysfunctional cognitive processes, such as worry, can be described as 'repetitive negative thinking' (RNT). Ehring et al. (2011) formulated three key characteristics of repetitive negative thinking: "(1a) 
the thinking is repetitive, (1b) it is at least partly intrusive, and (1c) it is difficult to disengage from" (p. 226, Ehring et al. 2011). Whereas the definition of rumination by NolenHoeksema et al. (2008) highlights the content of ruminative thoughts, the description by Ehring et al. (2011) focusses on the process of thinking. When combining these two leading definitions, depressive rumination can be described as dysfunctional cognitions about the causes and implications of negative emotions, which are repetitive, intrusive and difficult to disengage from.

Research has shown that rumination is related to the onset and duration of a depressive episode (Michalak et al. 2011; Nolen-Hoeksema 2000). Moreover, there are indications that rumination is more pronounced in chronically depressed patients compared to patients with non-chronic (i.e. shorter) depressive episodes (Wiersma et al. 2011). Rumination is related to the experienced quality of life in depressed patients, above and beyond depressive symptoms (Kuehner and Buerger 2005). Reducing rumination in chronically depressed patients is therefore a valuable treatment outcome.

Previous research concluded that MBCT significantly reduces rumination in recurrently depressed patients (Geschwind et al. 2012; van Aalderen et al. 2011; van Vugt et al. 2012). In addition, several studies found that rumination mediates the effect of MBCT on depressive symptoms (Shahar et al. 2010; van Aalderen et al. 2011) which would support the assumption that rumination is a working mechanisms of MBCT. Surprisingly, given the important role of rumination in chronic depression (Torpey and Klein 2008) and its important influence on the duration of episodes (Nolen-Hoeksema et al. 2008) only few studies investigated the effect of MBCT on rumination in chronic or treatmentresistant depression.

Whereas one randomized controlled trial (Cladder-Micus et al. 2018) and a pilot study (Eisendrath et al. 2008) found effects of MBCT on rumination in chronic and treatment resistant-depression, another randomized controlled trial found no effect (Eisendrath et al. 2016). In fact, a systematic review by van der Velden et al. (2015) concluded that the results on the effects of MBCT on rumination are inconsistent. Importantly, van der Velden et al. (2015) suggest to combine measures of self-report with laboratory measures of rumination increase or knowledge about the effects of $\mathrm{MBCT}$ on rumination. In sum, the effect of MBCT on rumination is currently not well understood, and data in chronic or treatment-resistant depression is largely lacking. As this group consists of patients with especially high disease burden, finding effective ways to reduce rumination might significantly improve their well-being.

Typically, rumination is assessed by validated questionnaires, such as the widely used Ruminative Response Scale (Nolen-Hoeksema and Morrow 1991). To specifically investigate levels of state rumination (i.e. rumination during a specific period of time) rather than trait rumination (i.e. the general tendency to ruminate), previous research used measures such as the 'brief state rumination inventory' (BSRI; Marchetti et al. 2018) or visual analogue scales (VAS) (Ciesla et al. 2012; Hilt and Pollak 2012; Key et al. 2008; Puterman et al. 2010; Zoccola and Dickerson 2012). All of these measures rely on self-report and require insight in one's own (typical) cognitive reaction. Although self-report has several advantages, it is not always in accordance with actual behaviour (Wilson and Dunn 2004) and it therefore has been argued that psychological studies should place a greater emphasis on observing behaviour instead of solely relying on self-report (Baumeister et al. 2007). Furthermore, most measures of state rumination rely on retrospective recall, a process that is typically biased in depressed individuals (Ben-Zeev et al. 2009; Gotlib and Joormann 2010; Solhan et al. 2009). To be able to disentangle effects of memory bias from other cognitive processes, the distinction between 'on-line' and 'off-line' measures can be helpful. On-line measures assess behaviour concurrent to task performance, whereas off-line measures assess behaviour after it happened (Veenman 2011). Examples of on-line measures are 'think-aloud protocols' to investigate metacognition in mathematical problem solving (Azevedo et al. 2010; Jacobse and Harskamp 2012) and reaction time paradigms to assess interpretation bias in anxiety disorders (Garner et al. 2006; Hirsch et al. 2006). Most of the currently available measures of rumination can be categorized as off-line measures, because they require participants to retrospectively indicate their level of rumination.

The Breathing Focus Task (BFT) is an on-line behavioural task that assesses repetitive negative thinking. The task was originally developed by Borkovec and colleagues (Borkovec et al. 1983) and has been adapted by Hirsch and colleagues (Hayes et al. 2010; Hirsch et al. 2009) to assess worry in general anxiety disorder. Recently, the BFT has been utilized to assess rumination in a student sample (Southworth et al. 2017). During the BFT participants are asked to focus on their breathing and to report distracting thoughts. In the original task also a worry or negative mood induction is used (e.g. Hirsch et al. 2009). Because the task assesses the number of dysfunctional negative thoughts during task performance, it minimizes influences of retrospective biases and reduces response bias. Although the advantages of an on-line task above using questionnaires are especially relevant in clinical samples, the BFT has not been used in clinically depressed individuals.

The overall goal of the current research was to investigate the effects of MBCT on rumination with an on-line behavioural measure in chronically, treatment-resistant depressed patients. Because the BFT has not been used in this population, the first aim was to investigate whether chronically, treatment-resistant depressed patients show 
more negative thought intrusions on the BFT compared with never-depressed individuals, as would be expected from previous studies using self-report. Therefore, thought intrusions of the BFT and effects on mood were compared between a never-depressed sample and a chronically, treatment-resistant depressed patient sample in a cross-sectional design. Secondly, in the same patient sample, the effect of MBCT on rumination measured with the BFT was examined in an randomized-controlled trial (RCT) comparing MBCT with treatment-as-usual. We hypothesized that MBCT would lead to a decrease in on-line behavioural rumination. A better understanding of the effects of MBCT on rumination in chronically, treatment-resistant depressed patients would allow more specific predictions about the effects of MBCT and therefore better patient care.

\section{Method}

This study is part of an RCT with 106 participants assessing the effects of MBCT versus treatment-as-usual (TAU) in chronic, treatment-resistant depression (Cladder-Micus et al. 2015, 2018, Dutch trial register [part of the Cochrane Collaboration], http://www.trialregister.nl: NTR4843). Patients were randomized to an 8-week MBCT training in combination with TAU (MBCT + TAU) or TAU alone. The procedure of the RCT regarding the clinical effectiveness is described in detail the published study protocol (Cladder-Micus et al. 2015) and is summarized below. The study protocol of the RCT was approved by the Medical Ethics Committee Arnhem-Nijmegen. Additionally, data of never-depressed controls were collected. The study protocol to test neverdepressed controls was considered as exempt from review by the Medical Ethics Committee Arnhem-Nijmegen.

\section{Participants and Procedure}

\section{Patients with Chronic, Treatment-Resistant Depression}

Participants were outpatients of a mental health clinic (Pro Persona) or an academic hospital (Radboud University Medical Center) in the Netherlands. Inclusion criteria were: (a) age $\geq 18$; (b) current depressive episode according to DSMIV criteria with a duration of $\geq 12$ months; (c) moderate to high levels of depressive symptoms (Inventory of Depressive Symptomatology-Self-Report, IDS-SR $\geq 21$ ); (d) previous psychological treatment during the current episode (defined as: $\geq 10$ sessions of Cognitive Behavioural Therapy or Interpersonal therapy ; or $<10$ sessions if discontinued because of patient's withdrawal); (e) at least one adequate trial of antidepressant medication during the current episode (defined as: appropriate doses of antidepressant medication for $\geq 4$ weeks; or patient's refusal to use medication contrary to the advice of a psychiatrist). Exclusion criteria were: (a) current psychotic symptoms; (b) lifetime bipolar disorder; (c) current alcohol or drug dependence; (d) recent electroconvulsive therapy ( $<3$ months ago); (e) current somatic disorder partly explaining depressive symptoms; (f) physical-, linguistic-, cognitive, or intellectual impairments which might interfere with participation in MBCT or assessments; and $(\mathrm{g})$ previous MBCT training. All patients gave written informed consent during a research interview. On baseline and post-treatment (MBCT + TAU,TAU), questionnaires and clinical interviews were administered (Cladder-Micus et al. 2015). Of the 106 participants a subsample of 86 participants (based on study entry ${ }^{1}$ ) was invited to additionally take part in a $1.5 \mathrm{~h}$ neuropsychological test battery including tasks on attention, memory, interpretation, cognitive control and rumination. There was no significant difference in baseline depressive symptoms between the whole sample $(\mathrm{N}=106)$ and the subsample $(\mathrm{n}=86)$ who was invited to take part in the experimental test battery, $t(104)=-0.89, p=0.37$. At the end of the test battery participants performed the BFT. Of 73 participants who agreed to perform the BFT at baseline, 62 participants (MBCT + TAU: 26, TAU: 36) also performed the task post-treatment. Patients who refused or agreed to take part in the neuropsychological test battery did not differ on levels of depressive symptoms, $t(84)=-0.01$, $p=0.99$. There was also no difference in depressive symptom levels between patients with or without missing data at post-treatment, $t(72)=-0.07, p=0.94$. Neuropsychological data collection took place between November 2013 and April 2016.

\section{Never-Depressed Controls}

Never-depressed control participants $(\mathrm{N}=108)$ were recruited from the community via flyers, websites and waitlists of mindfulness-based stress reduction (MBSR) courses provided for the general public. Exclusion criteria were: (a) current of lifetime depressive episode according to DSM-IV criteria; (b) current anxiety disorder according to DSM-IV criteria; (c) current or lifetime psychotic symptoms; (d) current alcohol or drugs dependence according to DSM-IV; (f) physical-, language-, cognitive-, or intellectual impairments which interfere with assessments; (g) previous mindfulness training or other mediation practice. We ensured that participants recruited via MBSR courses participated in the current study before attending the first MBSR session. Two individuals with excessive high scores on depressive symptoms (IDS-SR > 21) were excluded, leaving 106 participants for

\footnotetext{
${ }^{1}$ Data collection of the experimental test battery started while the RCT was ongoing. Therefore, the first 20 participants were not invited to perform the BFT.
} 
the analyses. The group was matched in age and gender to the patient sample. Interested individuals were telephonically screened for in- and exclusion criteria using the structured clinical interview for DSM-IV (SCID) axis I disorders (van Groenestijn et al. 1999). Before the neuropsychological test session, participants completed an online version of the questionnaires at home. The neuropsychological test session was identical for patients and never-depressed controls, except for two additional tasks that were added for the controls at the end of the session. These two tasks are not part of the current investigation. Participants gave written informed consent at the beginning of the neuropsychological test session and passive informed consent at the beginning of the online questionnaires. Participants received a gift card (€20) for their time and effort. Data collection of this sample took place between March 2014 and February 2016.

\section{Measures}

\section{Questionnaires}

Trait rumination Trait rumination was assessed with the extended version of the Ruminative Response Scale (RRSEXT; Raes and Hermans 2007; Raes et al. 2009). The RRSEXT distinguishes between two subscales: reflective pondering and brooding. The questionnaire consists of 26-items. Example items are 'I think about how alone I feel' or 'I think about why I always react the way I do'. Participants are asked to indicate the frequency of ruminative thoughts ( $1=$ almost never to $4=$ almost always $)$ when feeling down or depressed. Previous research reported appropriate reliability and validity (Schoofs et al. 2010). In the current study the reliability was high $(\alpha=0.87$ in patients; $\alpha=0.91$ in never-depressed controls).

Depressive symptoms Depressive symptoms were investigated with the Inventory of Depressive Symptomatology Self-Report (IDS-SR). The IDS-SR is a 30-item self report questionnaire and has good psychometric properties (Rush et al. 1996, 2006). The IDS-SR has previously been used in research on MBCT and has been shown to be sensitive to change (Geschwind et al. 2012). The reliability in the current study was appropriate $(\alpha=0.78$ in patients; $\alpha=0.71$ in never-depressed controls).

Mindfulness skills Mindfulness skills were assessed with the Five Facets Mindfulness Questionnaire (FFMQ; Baer et al. 2008). The FFMQ consists of 39 items which are divided in five subscales: observing, describing, acting with awareness, non-judging of inner experience and non-reactivity of inner experience. The Dutch version of the FFMQ has good psychometric properties (de Bruin et al. 2012; Veehof et al. 2011). In the current study, reliability of the total score was high ( $\alpha=0.82$ in patients; $\alpha=0.86$ in never-depressed controls). The reliability of the subscales was high ranging from $\alpha=0.79$ to $\alpha=0.89$, except for the subscale non-reactivity of inner experience, which showed lower reliability ( $\alpha=0.63$ in patients; $\alpha=0.70$ in never-depressed controls).

\section{Behavioural On-Line Assessment of Rumination: Breathing Focus Task}

The BFT (Borkovec et al. 1983; Hirsch et al. 2009) typically consists of two assessment phases and a worry induction (Hayes et al. 2010) or negative mood induction (Southworth et al. 2017). Due to ethical concerns when using a negative mood induction in a clinical sample, only the first assessment phase of the BFT was used in the current study. Participants were asked to focus on their breathing for five minutes, while noticing distracting thoughts (i.e. thought intrusions). During this period a computer generated tone sounded 12 times at random intervals of 20-30 s. After each tone participants were required to verbally report whether they were focused on their breathing or distracted by a thought intrusion. When distracted by a thought, participants reported a short word label (e.g. "cannot concentrate"), and classified the thought as negative, positive or neutral. When focused on their breathing participants responded by saying "breath" (Dutch: "adem"). The BFT task therefore assess intrusive thoughts 'on-line', i.e. during task performance. Participants were not explicitly informed that the task is designed to measure rumination. Prior to the 5-min breathing period, participants practiced to focus on their breathing (20 s) and practiced to respond to three tones while focusing on the breath (45 s). After the 5-min breathing period, participants filled in a self-report measure of state rumination consisting of a VAS (percentage of time spend ruminating [Dutch: "piekeren"], 1-100\%). Additionally, participants rated on three VAS the percentage of time they were able to focus on the breath $(0-100 \%)$, the percentage of time they were distracted by positive thoughts $(0-100 \%)$, and how difficult it was to focus on the breath (very difficult-not at all difficult). The number of negative thought intrusions (ranging from 0 to 12 ) reflects the individual state rumination of a participant. ${ }^{2}$ In case participants only reported the valence of an intrusion but no description, intrusions were still included in the scoring.

\footnotetext{
2 At the end of the BFT participants were asked to provide a more detailed description of the thoughts reported during the 5 min breathing period, which were audio-taped. A subsample of the detailed descriptions $(n=63)$ was rated by an independent assessor. The assessor blindly rated the descriptions as negative, positive or neutral. The correlation between the number of participant-rated and assessorrated negative thought intrusions was very high $(r=0.93, p<0.001)$. Analyses based on the assessor-ratings revealed comparable results and are available on request from the first author.
} 


\section{Mood Ratings}

Three VAS were used to assess sadness, anxiety and tension before and after the BFT. The scales ranged from 0 to 100 , with either sad, anxious, or tense on the right side and safe, relaxed, or happy on the left side.

\section{Interventions}

\section{$\mathrm{MBCT}+\mathrm{TAU}$}

MBCT was based on the manual described by Segal, Williams and Teasdale (2002) and consisted of 8 weekly $2.5 \mathrm{~h}$ sessions and 1 day of practice (day of silence). All mindfulness teachers were highly experienced in working with depressed patients and met the advanced criteria of the internationally agreed good practice guidelines of the UK Network for Mindfulness-based teachers (UK Network of Mindfulness-Based Teacher Trainers 2015). In conjunction with MBCT participants received TAU.

\section{TAU}

Treatments-as-usual was a naturalistic condition consisting of mental health care for depression, including antidepressant medication, psychological treatment, or support by a psychiatric nurse. At baseline the majority of patients used antidepressant medication (MBCT + TAU: $76.9 \%$; TAU: $80.6 \%$ ) and there were only minor changes in the number of patients who used antidepressant medication during posttreatment (MBCT: 65.4\%; TAU: 69.4\%). The mean number of therapy sessions by a psychologist during the study period were $\mathrm{M}=2.68(\mathrm{SD}=4.37)$ in the $\mathrm{MBCT}+\mathrm{TAU}$ condition and $\mathrm{M}=4.83(\mathrm{SD}=4.52)$ in the TAU condition.

\section{Statistical Analyses}

\section{Patients with Chronic, Treatment-Resistant Depression Versus Never-Depressed Controls}

Independent sample t-tests and $\chi^{2}$ tests were used to compare demographic variables between the groups. In case non-normality was observed based on visual inspection and Kolmogorov-Smirnov (K-S) tests, statistical tests not assuming a normal distribution (non-parametric tests, negative binominal regression analyses) were used. First, the total number of thought intrusions was compared between the groups, after which the number of negative thought intrusions, positive and neutral thought intrusions were investigated separately. Secondly, self-reported state rumination (VAS) was compared between the groups and its correlation with negative thought intrusion was computed. Third, effects of the BFT on mood were analyzed. Finally, Pearson and Spearman correlations between the number of negative thought intrusions and trait rumination, mindfulness skills and depressive symptoms were investigated. Effect sizes of non-parametric tests were calculated as: $r=z / \sqrt{N}$.

\section{MBCT + TAU Versus TAU}

We included all participants who completed the BFT at baseline and at post-treatment, irrespective whether participants attended all sessions of the MBCT. Independent sample t-tests and $\chi^{2}$ tests were used to compare demographic variables and baseline scores between the conditions (MBCT + TAU, TAU). The sum scores of all thought intrusions, negative intrusions, positive intrusions, and neutral intrusions were compared between the conditions by using mixed model ANOVAs. In line with previous research (Hayes et al. 2010; Hirsch et al. 2009), the number of negative intrusions was considered as the primary outcome. We also tested effects on self-reported state rumination (VAS). Furthermore, we computed Pearson correlations between change in negative thought intrusion from baseline to posttreatment and change in self-reported state rumination, trait rumination, depressive symptoms, and mindfulness skills.

\section{Results}

\section{Patients with Chronic, Treatment-Resistant Depression Versus Never-Depressed Controls}

The first aim of the study was to investigate whether neverdepressed control participants differed from chronically, treatment-resistant depressed patients on the BFT. The groups were comparable on age and gender, however the average level of education was higher in never-depressed controls, see Table 1.

\section{Breathing Focus Task}

Means, standard deviations and medians (as used in the nonparametric tests) of the group comparisons are displayed in Table 2 and graphically illustrated in Fig. 1. The total number of thought intrusions (irrespective of valence) was significantly higher in the patient group compared with the never-depressed control group. When including the level of education as a covariate in an ANCOVA, comparable results were found, $F(2,170)=41.03, p<0.001, \eta_{p}^{2}=0.19$. Data of thought intrusions were not normally distributed the neverdepressed control group (negative: $D(106)=0.37, p<0.001$; positive: $D(106)=0.26, p<0.001$; neutral: $D(106)=0.21$, $p<0.001)$.

Count data that are positively skewed are frequently analysed with Poisson regression analysis. However, this 
Table 1 Demographic characteristics and scores on questionnaires of patients with chronically, treatment-resistant depression and neverdepressed controls

\begin{tabular}{llll}
\hline & Patients $(\mathrm{N}=73)$ & $\begin{array}{l}\text { Never-depressed controls } \\
(\mathrm{N}=106)\end{array}$ & $t$-test/ $\chi^{2}$-test \\
\hline Demographics & & & $t(176)=0.56, p=0.58$ \\
$\quad$ Age, years & $46.9(10.12)$ & $45.9(12.5)$ & $\chi^{2}(1)=1.26, p=0.26$ \\
Gender, female/male & $43 \mathrm{~F} / 20 \mathrm{M}$ & $72 \mathrm{~F} / 34 \mathrm{M}$ & $t(171)=-4.67, p<0.001$ \\
$\quad$ Level of education (1-7) & $4.4(1.8)$ & $5.5(1.5)$ & $t(176)=34.52 . p<0.001$ \\
Questionnaires & & $5.39(3.82)$ & $t(173)=19.34, p<0.001$ \\
Depressive symptoms (IDS-SR) & $41.34(9.67)$ & $31.75(8.30)$ & $t(173)=-16.92, p<0.001$ \\
Trait rumination (RRS-EXT) & $58.11(9.54)$ & $139.98(15.99)$ & \\
Mindfulness skills (FFMQ) & $98.94(15.15)$ & & \\
\hline
\end{tabular}

Presented in mean (SD) unless otherwise specified

RRS-EXT Extended Version Ruminative Response Scale, FFMQ Five Facets Mindfulness Questionnaire, IDS-SR Inventory of Depressive Symptomatology Self-Report

Table 2 Group comparisons of patients with chronic, treatment-resistant depression and never-depressed controls

\begin{tabular}{|c|c|c|c|c|c|}
\hline & \multicolumn{2}{|c|}{ Patients $(\mathrm{N}=73)$} & \multicolumn{2}{|c|}{$\begin{array}{l}\text { Never-depressed } \\
\text { controls }(\mathrm{N}=106)\end{array}$} & \multirow[t]{2}{*}{ Test statistics } \\
\hline & $\mathrm{M}(\mathrm{SD})$ & Mdn & $\mathrm{M}(\mathrm{SD})$ & Mdn & \\
\hline \multicolumn{6}{|l|}{ Breathing focus task } \\
\hline Total intrusions & $6.08(3.05)$ & & $3.55(2.47)$ & & $t(176)=6.11, p<0.001, d=0.91$ \\
\hline Negative intrusions & $3.21(2.73)$ & 3.00 & $0.63(0.98)$ & 0.00 & $\begin{array}{l}\text { Neg. binomial regression: } \mathrm{B}=-1.59(\mathrm{SE}=0.20), p<0.001, \\
\operatorname{Exp}(\mathrm{B})=4.95,95 \% \mathrm{CI}[3.31 ; 7.38] ; \text { Mann-Whitney } \mathrm{U}: \\
U=1522, z=-7.2, p<0.001, r=0.54\end{array}$ \\
\hline Positive intrusions & $1.00(1.72)$ & 0.00 & $1.08(1.51)$ & 0.50 & $\begin{array}{l}\text { Neg. binomial regression: } \mathrm{B}=-0.05(\mathrm{SE}=0.21), p=0.80 \\
\operatorname{Exp}(\mathrm{B})=0.95,95 \% \text { CI }[0.62 ; 1.44] ; \text { Mann-Whitney } \mathrm{U} \text { : } \\
U=3969, z=0.491, p=0.62\end{array}$ \\
\hline Neutral intrusions & $1.81(1.99)$ & 1.00 & $1.84(1.89)$ & 1.00 & $\begin{array}{l}\text { Neg. binomial regression: } \mathrm{B}=-0.02(\mathrm{SE}=0.19), \mathrm{p}=0.92 \\
\operatorname{Exp}(\mathrm{B})=0.98,95 \% \text { CI }[0.67 ; 1.42] ; \text { Mann-Whitney } \mathrm{U} \text { : } \\
U=3875, z=0.179, p=0.86\end{array}$ \\
\hline \multicolumn{6}{|l|}{ Self-report (VAS) } \\
\hline Self-reported state rumination (\%) & $43.4(27.9)$ & 44.00 & $8.5(14.5)$ & 2.00 & $U=985, z=-8.04, p<0.001, r=0.61$ \\
\hline Time focused on breathing $(\%)$ & $49.2(25.2)$ & & $73.9(20.9)$ & & $t(169)=-6.94, p<0.001$ \\
\hline Difficulty to focus on breathing (1-100) & $58.3(28.2)$ & & $32.6(28.5)$ & & $t(169)=5.73, p<0.001$ \\
\hline Distracted by positive thoughts (\%) & $23.3(20.8)$ & & $25.3(26.9)$ & & $t(168)=-0.50, p=0.64$ \\
\hline
\end{tabular}

$M$ mean, $M d n$ median, $S D$ standard deviation, $V A S$ visual analogue scale

distribution assumes that the mean and the variance are equal which is not the case in the current sample (see Table 2). Negative binomial regression relaxes the strict mean-variance relationship of the Poisson regression. Independent negative binomial regression analyses were conducted for negative intrusions, positive intrusions, and neutral intrusion with group (never-depressed controls, patient group) as predictor. Results show that group significantly predicted the amount of negative thought intrusions but not the amount of positive or neutral thought intrusions (see Table 2). The incident risk ratio for negative thought intrusions was 4.59 times higher for the patient group compared to the neverdepressed control group. Including level of education as a predictor in the models only had a significant effect on the amount of neutral intrusions $\mathrm{B}=0.27, \mathrm{SE}=0.06, p<0.001$, $\operatorname{Exp}(B)=1.31,95 \%$ CI $[1.16 ; 1.49]$, which means that with each point increase in educational level the rate of neutral intrusions is expected to increase by a factor of 1.31 , when holding other variables constant. Results of Mann-Whitney U-tests are in accordance with these results. The number of negative thoughts in the patient group was significantly higher compared with the never-depressed controls $(p<0.001)$ with a large effect size of $r=-0.54$. In contrast, no differences in the number of positive $(p=0.62)$ or neutral thought intrusions $(p=0.86)$ were observed, see Fig. 1. 


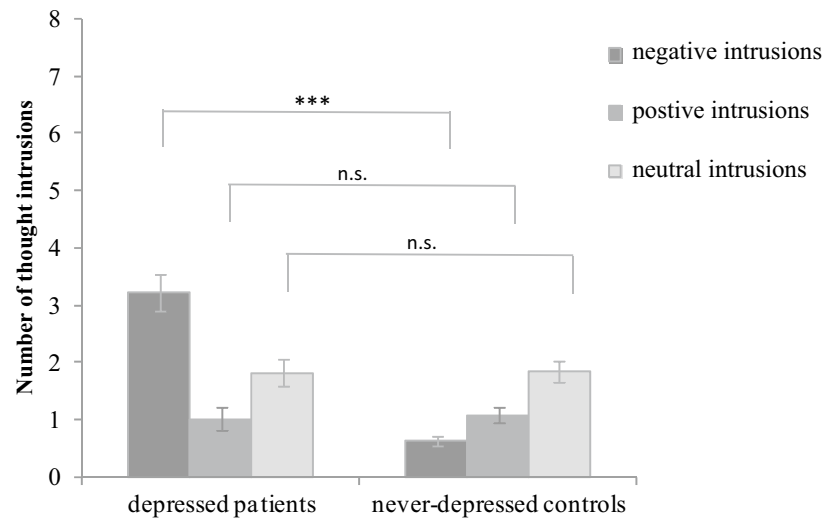

Fig. 1 Means of negative, positive, and neutral thought intrusions on the breathing focus task for both groups. Error bars display standard error of the mean, $* * * p<0.001$

Patients spent a significantly lower percentage of time focused on the breathing $(p<0.001)$ and found it more difficult to focus on the breathing $(p<0.001)$. On the self-report measure of rumination (VAS) patients reported higher rumination than never-depressed controls $(p<0.001)$ with a large effect size of $r=0.61$. There was no difference between the groups in the percentage of time they were distracted by positive thoughts.

\section{Effects on Mood}

Changes in mood (sadness, anxiety, tension) from pre to post BFT were investigated with Wilcoxon Signed Rank Tests, because data were not normally distributed. The means, standard deviations and medians of the mood ratings are displayed in Table 3. Depressed patients showed a significant increase in sadness ( $p=0.002, z=3.08, r=0.38$, medium to large effect) and no change in anxiety $(p=0.10)$ or tension $(p=0.83)$. In contrast, the never-depressed control group showed no change in sadness $(p=0.83)$ or anxiety $(p=0.06)$, but a significant decrease in tension, $p<0.001$, $z=-3.80, r=-0.37$, medium to large effect.

\section{Correlations}

Correlations between the measures are presented in Table 4 . The number of negative thought intrusions was significantly correlated with self-reported state rumination in both samples. However, only in the never-depressed sample but not in the depressed sample a significant correlation between negative thought intrusions and trait rumination was found. Likewise, only the never-depressed control sample showed a correlation between self-reported state rumination (VAS)

Table 3 Mood at pre and post BFT in patients with chronic, treatment-resistant depression and never-depressed controls

\begin{tabular}{|c|c|c|c|c|c|c|c|c|}
\hline & \multicolumn{4}{|l|}{ Patients } & \multicolumn{4}{|c|}{ Never-depressed controls } \\
\hline & \multicolumn{2}{|l|}{ Pre BFT } & \multicolumn{2}{|l|}{ Post BFT } & \multicolumn{2}{|l|}{ Pre BFT } & \multicolumn{2}{|l|}{ Post BFT } \\
\hline & $\mathrm{M}(\mathrm{SD})$ & Mdn & $\mathrm{M}(\mathrm{SD})$ & Mdn & $\mathrm{M}(\mathrm{SD})$ & Mdn & $\mathrm{M}(\mathrm{SD})$ & Mdn \\
\hline Sadness & $59.0(19.1)$ & 57.0 & $63.6(20.3)$ & 63.0 & $11.8(14.8)$ & 5.0 & $12.2(15.7)$ & 6.0 \\
\hline Anxiety & $39.2(24.0)$ & 39.0 & $43.5(24.1)$ & 50.0 & $6.0(11.3)$ & 1.0 & $6.1(10.1)$ & 0.0 \\
\hline Tension & $56.0(24.0)$ & 58.5 & $57.4(26.4)$ & 60.0 & 14.4 (18.6) & 8.0 & $10.1(15.5)$ & 2.0 \\
\hline
\end{tabular}

$B F T$ breathing focus task, $M$ mean, $S D$ standard deviation, $M d n$ median

Table 4 Correlations between different state and trait measures of rumination, depressive symptoms, sadness, and mindfulness skills in patients with chronic, treatment-resistant depression and never-depressed controls

\begin{tabular}{llllllll} 
& \multicolumn{7}{l}{ Patients with chronic, treatment-resistant depression } \\
\cline { 2 - 8 } & $\begin{array}{l}\text { IDS- } \\
\text { SR }\end{array}$ & $\begin{array}{l}\text { RRS- } \\
\text { EXT }\end{array}$ & $\begin{array}{l}\text { RRS- } \\
\text { brooding }\end{array}$ & $\begin{array}{l}\text { RRS- } \\
\text { reflective }\end{array}$ & FFMQ & BFT & $\begin{array}{l}\text { VAS } \\
\text { rumination }\end{array}$ \\
\cline { 2 - 8 } RRS-SR & - & & & & & & \\
RRS-EXT & $.35^{* *}$ & - & & & & & \\
RRS-brooding & .21 & $.77^{* *}$ & - & & & & \\
RRS-reflective & .04 & $.68^{* *}$ & $.34^{* *}$ & - & & & \\
FFMQ & $-.33^{* *}$ & -.22 & $-.31^{* *}$ & .19 & - & & \\
BFT & .09 & -.06 & -.09 & -.08 & $-.26^{*}$ & - & \\
VAS-rumination & $-.01^{\mathrm{a}}$ & $-.11^{\mathrm{a}}$ & $-.17^{\mathrm{a}}$ & $-.002^{\mathrm{a}}$ & $-.31^{*}$ a & $.64^{* *}$ a & - \\
VAS-sadness $^{1}$ & $.32^{* *}$ & .09 & .003 & -.03 & $-.33^{* *}$ & $.24^{*}$ & .04 \\
\hline
\end{tabular}

\begin{tabular}{|c|c|c|c|c|c|c|}
\hline $\begin{array}{l}\text { IDS- } \\
\text { SR }\end{array}$ & $\begin{array}{l}\text { RRS- } \\
\text { EXT }\end{array}$ & $\begin{array}{l}\text { RRS- } \\
\text { brooding }\end{array}$ & $\begin{array}{l}\text { RRS- } \\
\text { reflective }\end{array}$ & FFMQ & BFT & $\begin{array}{l}\text { VAS } \\
\text { rumination }\end{array}$ \\
\hline \multicolumn{7}{|l|}{-} \\
\hline $.49^{* *}$ & - & & & & & \\
\hline $.55^{* *}$ & $.84 * *$ & - & & & & \\
\hline $.26^{*}$ & $.83 * *$ & $.57 * *$ & - & & & \\
\hline$-.42 * *$ & $-.23 *$ & $-.31 * *$ & -.04 & - & & \\
\hline $.13^{\mathrm{a}}$ & $.41 * * \mathrm{a}$ & $.40 * * \mathrm{a}$ & $.35 * * \mathrm{a}$ &.$-20 * \mathrm{a}$ & - & \\
\hline $.29 * * \mathrm{a}$ & $.44 * * \mathrm{a}$ & $.41 * *$ a & $.40 * * \mathrm{a}$ & $-.38 * * \mathrm{a}$ & $.46 * * a$ & - \\
\hline $.23^{* a}$ & $.33 * a$ & $.33 * \mathrm{a}$ & $.29 * * \mathrm{a}$ & $-.37 * * \mathrm{a}$ & $.31 * \mathrm{a}$ & $.39 * * a$ \\
\hline
\end{tabular}

$* \mathrm{p}<0.05, * * \mathrm{p}<0.001$

${ }^{\text {a }}$ Spearman correlation

${ }^{1}$ Prior to BFT

BFT Breathing Focus Task, RRS-EXT Extended Version Ruminative Response Scale, FFMQ Five Facets Mindfulness Questionnaire, IDS-SR Inventory of Depressive Symptomatology Self-Report, VAS Visual Analogue Scale 
Table 5 Characteristics per condition on baseline

\begin{tabular}{llll}
\hline & MBCT + TAU $(\mathrm{n}=26)$ & TAU $(\mathrm{n}=36)$ & $t$-test $/ \chi^{2}$-test \\
\hline Demographic characteristics & & & $t(71)=-0.75, p=0.455$ \\
Age, years & $47.9(9.7)$ & $46.1(10.5)$ & $21 \mathrm{~F} / 18 \mathrm{M}$ \\
Gender, female/male & $21 \mathrm{~F} / 13 \mathrm{M}$ & $\chi^{2}(1)=0.466, p=0.50$ \\
Level of education (1-7) & $4.2(1.9)$ & $4.4(1.8)$ & $t(66)=0.44, p=0.66$ \\
Trait measures & & $42.50(9.30)$ & $t(71)=0.92, p=0.36$ \\
Depressive symptoms (IDS-SR) & $40.39(10.20)$ & $59.06(9.31)$ & $t(68)=0.99, p=0.33$ \\
Trait rumination (RRS-EXT) & $56.80(9.70)$ & $96.95(14.39)$ & $t(68)=-1.09, p=0.28$ \\
Mindfulness skills (FFMQ) & $100.91(16.08)$ & & $t .05(2.85)$ \\
State measures & & $41.5(26.3)$ & $t(71)=-0.601, p=0.55$ \\
Negative intrusions (BFT) & $3.44(2.59)$ & $57.5(18.0)$ & $t(63)=-0.598, p=0.55$ \\
Self-reported state rumination (VAS $\%)$ & $45.6(30.0)$ & $39.9(22.9)$ & $t(67)=-1.02, p=0.31$ \\
Sadness (VAS) & $62.4(21.5)$ & $55.3(22.6)$ & $t(67)=0.004, p=0.99$ \\
Anxiety (VAS) & $39.9(30.0)$ & $t(67)=-0.52, p=0.60$ \\
Tension (VAS) & $58.4(26.9)$ & & \\
\hline
\end{tabular}

Presented in mean (SD) unless otherwise specified

$B F T$ Breathing Focus Task, FFMQ Five Facets Mindfulness Questionnaire, IDS-SR Inventory of Depressive Symptomatology Self-Report, $M B C T$ Mindfulness-based cognitive therapy, RRS-EXT Extended Version Ruminative Response Scale, TAU treatment-as-usual, VAS Visual Analogue Scale

${ }^{1}$ Based on Dutch classification system according to Verhage (1964): 1-2=low educational level (primary school; some secondary education), 3-5 = medium educational level (secondary education, low to medium level), 6-7 =high educational level (secondary education, high level; college degree; university degree)

and trait rumination whereas the depressed patient sample showed no significant correlation between the state and trait measures. In both samples the number of negative thought intrusions was significantly negatively correlated with mindfulness skills.

In neither of the two samples, the number of negative thought intrusions was correlated with depressive symptoms. By comparison, the self-reported state rumination measure (VAS) was significantly correlated with depressive symptoms in the never-depressed control sample but not in the depressed sample.

\section{MBCT + TAU Versus TAU}

This paragraph describes analyses related to the second- and main-aim of the study: to investigate whether MBCT influences rumination assessed with the BFT. On baseline there were no differences between the conditions (MBCT + TAU, TAU) on demographic variables and baseline scores, see Table 5.

\section{Breathing Focus Task}

In order to test whether MBCT + TAU resulted in a reduction of the total number of thought intrusions on the BFT compared with TAU, a mixed model ANOVA was conducted on the total number of thought intrusions (irrespective of valence) with time (baseline, post-treatment) as within-subject factor and condition (MBCT + TAU, TAU) as between-subject factor. Results showed a significant main effect of time, $F(1,60)=20.00, p<0.001, \eta_{p}^{2}=0.25$ and a significant time*condition interaction, $F(1,60)=6.46$, $p=0.014, \eta_{p}^{2}=0.097$. This means that both conditions showed a reduction in total thought intrusions from baseline to post-treatment, and that this decrease was larger in the MBCT + TAU condition than in the TAU condition, see Fig. 2.

Secondly, mixed model ANOVA's with time (baseline, post-treatment) as within-subject factor and condition (MBCT + TAU, TAU) as between-subject factor were conducted for each valence (negative, positive, neutral) separately. There was a main effect of time on negative thought intrusions, $F(1,60)=22.17, p<0.001, \eta_{p}^{2}=0.27$ and, more importantly, a significant interaction between time and condition, $F(1,60)=5.04, p=0.03, \eta_{p}^{2}=0.08$, with MBCT + TAU participants showing a larger decrease in negative thoughts than participants receiving TAU, see Fig. 2. We included change in depressive symptoms during MBCT + TAU or TAU (IDS post score-IDS pre score) as covariate in the analysis to assess independence of clinical improvement. When including change in depressive symptoms in the model, comparable results for the time* condition interaction were found, $F(1,58)=4.23$, $p=0.04, \eta_{p}^{2}=0.07$, indicating that the decrease in negative thought intrusions was independent of change in depressive symptoms. No main effect of time was found 


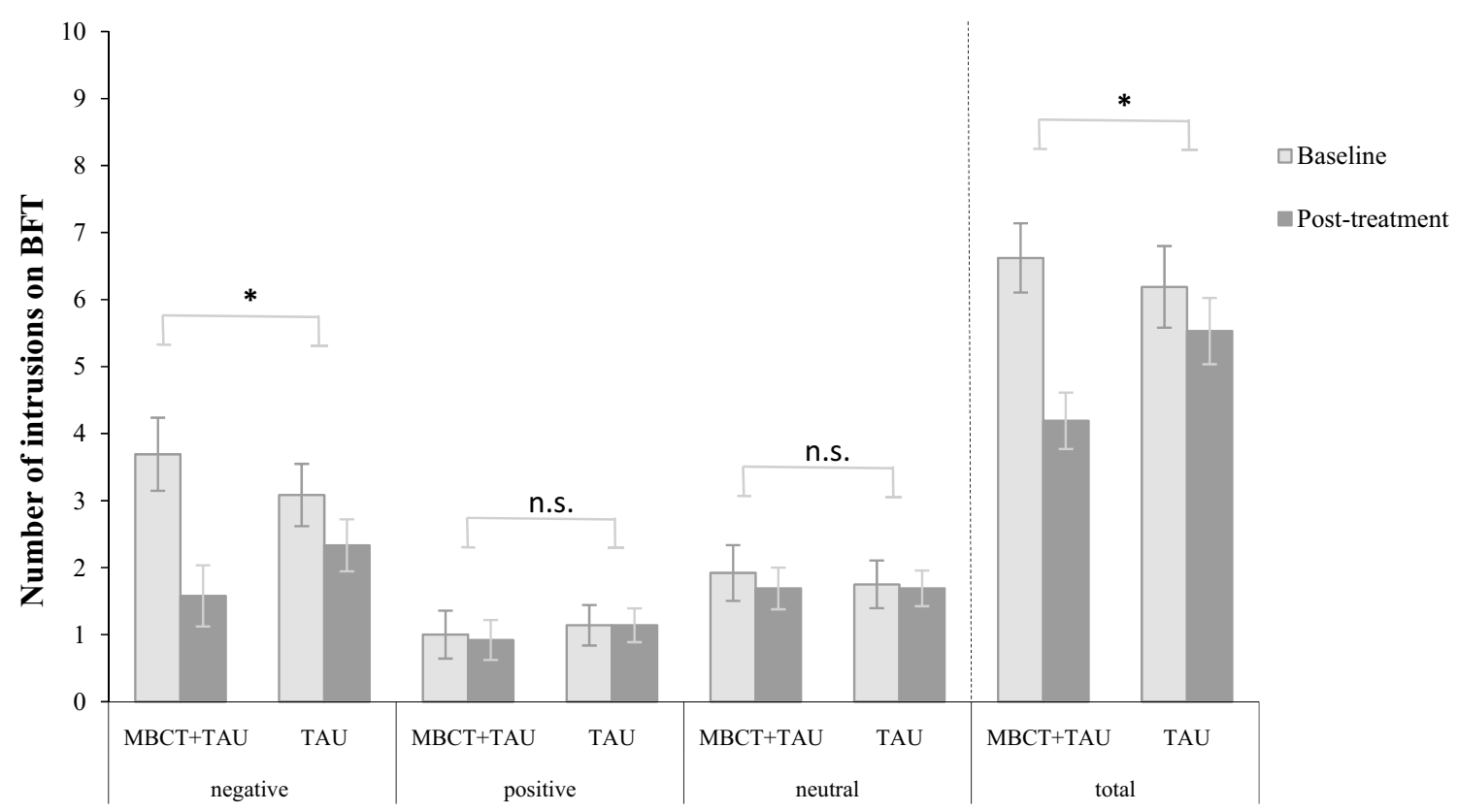

Fig. 2 Mean number of negative, positive, and neutral thoughts during the breathing focus task per condition on baseline and post-treatment. The total score is the sum of all thought intrusions. Error bars represent standard errors. BFT breathing focus task, $M B C T$ Mindfulness-based cognitive therapy, $T A U$ treatment-as-usual, $n s$ non significant, $* p<0.05$ for positive, $F(1,60)=0.024, p=0.88, \eta_{p}^{2}=0.00$ or neutral thought intrusions, $F(1,60)=0.33, p=0.57, \eta_{p}^{2}=0.01$, and no significant time*condition interactions were observed (positive: $F(1,60)=0.024, p=0.88, \eta_{p}^{2}=0.00$; neutral: $\left.F(1,60)=0.12, p=0.73, \eta_{p}^{2}=0.002\right)$, see Fig. 2 . Taken together, these results indicate that participants of the MBCT + TAU condition showed a larger decrease in negative thought intrusions than those of the TAU condition, whereas no change over time or differences between the conditions on positive or neutral thought intrusions were observed.

There was a main effect of time on the percentage of time participants reported to be able to focus on the breathing, $F(1,57)=13.95, p<0.001$, but no time *condition interaction, $F(1,57)=2.29, p=0.14$. Specifically, the percentage of time participants were able to focus on the breathing increased from baseline to post-treatment, however no significant differential effect for TAU ( $M_{\text {baseline }}=48.68, S D_{\text {baseline }}=25.15$, $\left.M_{\text {post-treatment }}=54.82, S D_{\text {post-treatment }}=24.81\right)$ or MBCT + TAU $\left(M_{\text {baseline }}=48.68, S D_{\text {baseline }}=27.37\right.$, $M_{\text {post-treatment }}=63.20, S D_{\text {post-treatment }}=19.57$ ) was found. There were no main or interaction effects regarding the self-reported difficulty to focus on the breathing or the time being distracted by positive thoughts (all $F<1$, $p>0.05)$.

\section{Effects on Self-Reported State Rumination}

To test whether MBCT + TAU resulted in a reduction of self-reported state rumination (VAS), a mixed model ANOVA with time (baseline, post-treatment) as within-subject variable and condition $(\mathrm{MBCT}+\mathrm{TAU}$, TAU) as between-subject variable was conducted. There was a significant effect of time, $F(1,57)=12.5$, $p<0.001, \eta_{p}^{2}=0.18$, but no time*condition interaction, $F(1,57)=2.73, p=0.10, \eta_{p}^{2}=0.05$, indicating that both conditions decreased in self-reported state rumination (MBCT + TAU: $M_{\text {baseline }}=47.12, S D_{\text {baseline }}=30.11$, $M_{\text {post-treatment }}=27.68, S D_{\text {post-treatment }}=21.89 ;$ TAU: $M_{\text {baseline }}=43.53, S D_{\text {baseline }}=26.71, M_{\text {post-treatment }}=36.47$, $S D_{\text {post-treatment }}=24.93$ ), but that there was no significant differential effect of condition.

\section{Correlations}

Change in negative thought intrusions from baseline to post-treatment was significantly positively correlated with change in self-reported state rumination in both conditions (MBCT+TAU: $r=0.44, p=0.03$; TAU: $r=0.51, p=0.002$ ). In neither of the conditions, change in negative thought intrusions from baseline to post-treatment was correlated with change in depressive symptoms (MBCT + TAU $r=0.22$, $p=0.29$; TAU: $-0.09, p=0.58)$, change in trait rumination 
(MBCT+TAU: $r=-0.14, p=0.51$; TAU: $r=0.12, p=0.49$ ) or change in mindfulness skills (MBCT $+\mathrm{TAU}: r=-0.09$, $p=0.68$; TAU: $r=-0.14, p=0.43$ ).

\section{Discussion}

The first aim of the current research was to investigate whether the BFT could be used as an on-line behavioural measure to assess rumination in chronically, treatment resistant depressed patients. Compared with never-depressed individuals from the community, patients with chronic, treatment-resistant depression reported significantly more negative thought intrusions on the BFT. Importantly, the groups did not differ in the number of positive or neutral thought intrusions, indicating that the difference between patients and controls was valence-specific. The number of negative thought intrusions significantly correlated with a more traditional measure of state rumination (i.e. VAS). As expected based on previous results (Hayes et al. 2010), the group of chronically, treatment-resistant depressed patients showed an increase in sadness after performing the BFT.

In both patients and never-depressed individuals rumination assessed with the BFT was correlated with sad mood, but not with depressive symptoms. By comparison, the selfreport state rumination measure (VAS) showed a significant correlation with sad mood and depressive symptoms in the never-depressed sample. This might indicate that the BFT shows less overlap with depressive symptoms than the selfreport measure of rumination. There is a need for state rumination measures that are independent of depressive symptoms because this would allow testing specific hypotheses about the effects of interventions on rumination and depressive symptoms (LeMoult et al. 2013). The BFT might partly fulfil this need. Interestingly, only in the never-depressed control sample but not in the depressed sample, the measures of state and trait rumination were significantly correlated. It is unlikely that this is due to ceiling effects, because the variation in scores was larger in the depressed sample than in the group of never-depressed controls. Previous research found that state and trait measures of rumination were not correlated in college students (LeMoult et al. 2013). It is therefore surprising that we found a correlation within the never-depressed sample but not within the patient sample. Our findings indicate that the relationship between the general tendency to ruminate and state rumination might be different for chronically, treatment-resistant depressed patients compared to healthy individuals.

The second- and main-aim of this study was to investigate whether MBCT influences rumination in patients with chronic, treatment-resistant depression. The results show that participants of MBCT + TAU compared with TAU show a decrease of negative thought intrusions on the BFT.
No changes of positive or neutral thought intrusions were observed. As expected, this reduction in negative thought intrusions on the BFT was significantly correlated with a more traditional self-report measure of state rumination (i.e. VAS). In contrast, no effect of MBCT + TAU compared with TAU on self-reported state rumination (VAS) was observed, which might indicate that the BFT could be a more sensitive measure than a VAS to assess state rumination.

Against our expectations, we found no correlations between the reduction of negative thought intrusions and change in trait rumination, depressive symptoms or mindfulness skills. That there was no correlation between the BFT and (change in) trait rumination might be explained by the fact that the BFT mostly focusses on the intrusiveness of ruminative thoughts, which was described as one of the key characteristics of RNT (Ehring et al. 2011). In contrast, the RRS focuses more on the content of ruminative thoughts. To further explore the relationships and differences between state (or on-line) and trait measures (often off-line) of rumination, it might be helpful to consider rumination as a coping mechanism to deal with negative emotions (NolenHoeksema et al. 1994). Lazarus (1993) argued that coping has trait and state aspects that are not necessarily identical. State coping styles might show more intraindividual change because contextual factors are of the greater influence. The general tendency to ruminate (i.e. trait) might be more stable than the use of rumination (i.e. state) in different situations. In the current sample we found changes in trait rumination (Cladder-Micus et al. 2018) and state rumination (the current paper) due to MBCT, which interestingly seem to be largely unrelated. Furthermore, the inconsistencies might be due to the fact that trait rumination, mindfulness skills, and depressive symptoms were assessed with questionnaires, whereas rumination was assessed with a behavioural task. Different measurement methods tend to show limited correlation whereas two measures using the same method have a higher chance of correlating significantly (Podsakoff et al. 2003). However, the absence of the correlations are puzzling and need further investigation.

\section{Strengths, Limitations and Future Research}

This paper describes the first randomized controlled trial showing that rumination assessed with a on-line, behavioural measure changes due to MBCT in chronically, treatment-resistant depressed patients. This result is in line with the theoretical model of MBCT, which proposes that MBCT influences depressive rumination (Segal et al. 2012). The current study thereby provides further information about the specific effects of MBCT, which was formulated as an important research goal in the field of mindfulness research (Dimidjian and Segal 2015). The current results are in line 
with the impaired disengagement hypothesis (Koster et al. 2011) which states that prolonged rumination is partly due to impaired attentional disengagement from negative information. The combination of greater awareness of thoughts and higher attentional control might increase the ability to shift attention and thereby to disengage from ruminative thoughts (Koster et al. 2011). One of the strengths of on-line measures is that the results are less influenced by memory biases during retrospective recall (Veenman 2011). Furthermore, although one could argue that even scores of the BFT are based on self-report, response biases are diminished because the participant is not explicitly asked whether he or she is ruminating. Therefore, the BFT provides a more direct reflection of change in rumination compared with studies using off-line measures, as for example questionnaires. The original BFT includes a worry or negative mood induction. Our results show that the BFT can be used in depressed patients without including an induction, which shortens the procedure and reduces ethical concerns in severely depressed patients. Although the task asks for a certain level of introspection and insight in one's own cognitive processes, our results show that chronically depressed patients are able to perform the task with the standard amount of practice. The BFT could therefore be a valuable instrument for future research on rumination, especially if one is interested in effects independent of memory biases. This could be especially valuable in research on working mechanisms of treatments.

Of course the current study is not without limitations. First of all, using the BFT as a measure of depressive rumination is a new approach which needs further validation. Previous research used the BFT as a measure of rumination in student samples (Southworth et al. 2017). Our findings underscore the need to further validate the BFT in clinical depressed samples because the relationship between state (on-line) measures of rumination and trait measures of rumination seems to be different for never-depressed individuals and chronically depressed patients. Secondly, a standardized scoring procedure for the BFT should be developed to allow direct comparisons between studies. In line with studies published by the developers of the BFT (Borkovec et al. 1983; Hirsch et al. 2009), we used the number of negative thought intrusions. However, alternative scorings have been published (Southworth et al. 2017). Third, it is important to realize that our findings are based on a pre-post design. Therefore the temporal order of change in rumination and depressive symptoms remains unclear (Kazdin 2007) and should be investigated in future research. Furthermore, it is important to keep in mind that the current study provides insights about the effects of MBCT for patients with chronic, treatment-resistant depression. Whether comparable results are observed in mildly depressed or remitted patients should be assessed in future research. Finally, the BFT not only requires sufficient insight into one's own cognitive processes, but also the willingness to share these thoughts. Especially responses of never-depressed participants, who are not used to discuss personal thoughts with a mental health professional, could be influenced by social desirability.

\section{Conclusion}

The current study shows that MBCT affects state rumination assessed with an on-line behavioural measure in chronically, treatment-resistant depressed patients. It therefore adds evidence to the theoretical assumption that MBCT reduces depressive rumination (Segal et al. 2012).

Acknowledgement A.E.M. Speckens is founder and clinical director of the Radboudumc Centre for Mindfulness. She is a mindfulness trainer and occasionally receives payment for training workshops and presentations related to mindfulness-based cognitive therapy. A.E.M. Speckens donates all such fees to Radboud University Medical Center.

Funding The RCT was partly funded by a Grant of Fonds Psychische Gezondheid, the Netherlands Foundation for Mental Health (Grant Number 2011 6630).

\section{Compliance with Ethical Standards}

Conflict of interest M.B. Cladder-Micus, J. Spijker, E.S. Becker, and J.N. Vrijsen declare they have no potential conflict of interest.

Ethical Approval All procedures performed in studies involving human participants were in accordance with the ethical standards of the institutional and/or national research committee and with the 1964 Helsinki declaration and its later amendments or comparable ethical standards.

Informed Consent All participants gave informed consent.

Animal Rights No animal studies were carried out by the authors for this article.

Open Access This article is distributed under the terms of the Creative Commons Attribution 4.0 International License (http://creativeco mmons.org/licenses/by/4.0/), which permits unrestricted use, distribution, and reproduction in any medium, provided you give appropriate credit to the original author(s) and the source, provide a link to the Creative Commons license, and indicate if changes were made.

\section{References}

Azevedo, R., Moos, D. C., Johnson, A. M., \& Chauncey, A. D. (2010). Measuring cognitive and metacognitive regulatory processes during hypermedia learning: Issues and challenges. Educational Psychologist, 45(4), 210-223.

Baer, R. A., Smith, G. T., Lykins, E., Button, D., Krietemeyer, J., Sauer, S., et al. (2008). Construct validity of the five facet mindfulness questionnaire in meditating and nonmeditating samples. Assessment, 15(3), 329-342. 
Baumeister, R. F., Vohs, K. D., \& Funder, D. C. (2007). Psychology as the science of self-reports and finger movements: Whatever happened to actual behavior? Perspectives on Psychological Science, 2(4), 396-403.

Ben-Zeev, D., Young, M. A., \& Madsen, J. W. (2009). Retrospective recall of affect in clinically depressed individuals and controls. Cognition and Emotion, 23(5), 1021-1040.

Borkovec, T. D., Robinson, E., Pruzinsky, T., \& DePree, J. A. (1983). Preliminary exploration of worry: Some characteristics and processes. Behaviour Research and Therapy, 21(1), 9-16.

Ciesla, J. A., Reilly, L. C., Dickson, K. S., Emanuel, A. S., \& Updegraff, J. A. (2012). Dispositional mindfulness moderates the effects of stress among adolescents: Rumination as a mediator. Journal of Clinical Child and Adolescent Psychology, 41(6), $760-770$

Cladder-Micus, M. B., Speckens, A. E., Vrijsen, J. N., Donders, T., Becker, A. R., E. S., \& Spijker, J. (2018). Mindfulness-based cognitive therapy for patients with chronic, treatment-resistant depression: A pragmatic randomized controlled trial. Depression and anxiety, 35(10), 914-924.

Cladder-Micus, M. B., Vrijsen, J. N., Becker, E. S., Donders, R., Spijker, J., \& Speckens, A. E. (2015). A randomized controlled trial of Mindfulness-Based Cognitive Therapy (MBCT) versus treatment-as-usual (TAU) for chronic, treatment-resistant depression: Study protocol. BMC Psychiatry, 15(1), 1-8.

de Bruin, E. I., Topper, M., Muskens, J. G., Bögels, S. M., \& Kamphuis, J. H. (2012). Psychometric properties of the Five Facets Mindfulness Questionnaire (FFMQ) in a meditating and a nonmeditating sample. Assessment, 19(2), 187-197.

Dimidjian, S., \& Segal, Z. V. (2015). Prospects for a clinical science of mindfulness-based intervention. American Psychologist, 70(7), 593.

Ehring, T., \& Watkins, E. R. (2008). Repetitive negative thinking as a transdiagnostic process. International Journal of Cognitive Therapy, 1(3), 192-205.

Ehring, T., Zetsche, U., Weidacker, K., Wahl, K., Schönfeld, S., \& Ehlers, A. (2011). The Perseverative Thinking Questionnaire (PTQ): Validation of a content-independent measure of repetitive negative thinking. Journal of behavior therapy and experimental psychiatry, 42(2), 225-232.

Eisendrath, S. J., Delucchi, K., Bitner, R., Fenimore, P., Smit, M., \& McLane, M. (2008). Mindfulness-based cognitive therapy for treatment-resistant depression: A pilot study. Psychotherapy and Psychosomatics, 77(5), 319-320. https://doi.org/10.1159/00014 2525.

Eisendrath, S. J., Gillung, E., Delucchi, K. L., Segal, Z. V., Nelson, J. C., McInnes, L. A., et al. (2016). A randomized controlled trial of mindfulness-based cognitive therapy for treatment-resistant depression. Psychotherapy and Psychosomatics, 85(2), 99-110.

Garner, M., Mogg, K., \& Bradley, B. P. (2006). Fear-relevant selective associations and social anxiety: Absence of a positive bias. Behaviour Research and Therapy, 44(2), 201-217.

Geschwind, P., van Os, H., \& Wichers (2012). Efficacy of mindfulnessbased cognitive therapy in relation to prior history of depression: Randomised controlled trial. The British Journal of Psychiatry, 201(4), 320-325. https://doi.org/10.1192/bjp.bp.111.104851.

Gotlib, I. H., \& Joormann, J. (2010). Cognition and depression: Current status and future directions. Annual Review of Clinical Psychology, 6(1), 285-312. https://doi.org/10.1146/annurev.clinpsy.12120 8.131305 .

Harvey, A. G., Watkins, E., Mansell, W., \& Shafran, R. (2004). Cognitive behavioural processes across psychological disorders. Oxford: Oxford University Press.

Hayes, S., Hirsch, C. R., Krebs, G., \& Mathews, A. (2010). The effects of modifying interpretation bias on worry in generalized anxiety disorder. Behaviour Research and Therapy, 48(3), 171-178. https ://doi.org/10.1016/j.brat.2009.10.006.

Hilt, L. M., \& Pollak, S. D. (2012). Getting out of rumination: Comparison of three brief interventions in a sample of youth. Journal of Abnormal Child Psychology, 40(7), 1157-1165.

Hirsch, C., Clark, D., \& Mathews, A. (2006). Imagery and interpretations in social phobia: Support for the combined cognitive biases hypothesis. Behavior Therapy, 37(3), 223-236. https://doi. org/10.1016/j.beth.2006.02.001.

Hirsch, C. R., Hayes, S., \& Mathews, A. (2009). Looking on the bright side: Accessing benign meanings reduces worry. Journal of Abnormal Psychology, 118(1), 44-54. https://doi.org/10.1037/ a0013473.

Jacobse, A. E., \& Harskamp, E. G. (2012). Towards efficient measurement of metacognition in mathematical problem solving. Metacognition and Learning, 7(2), 133-149.

Kazdin, A. E. (2007). Mediators and mechanisms of change in psychotherapy research. Annual Review of Clinical Psychology, $3,1-27$.

Key, B. L., Campbell, T. S., Bacon, S. L., \& Gerin, W. (2008). The influence of trait and state rumination on cardiovascular recovery from a negative emotional stressor. Journal of Behavioral Medicine, 31(3), 237-248.

Koster, E. H., De Lissnyder, E., Derakshan, N., \& De Raedt, R. (2011). Understanding depressive rumination from a cognitive science perspective: The impaired disengagement hypothesis. Clinical Psychology Review, 31(1), 138-145.

Kuehner, C., \& Buerger, C. (2005). Determinants of subjective quality of life in depressed patients: The role of self-esteem, response styles, and social support. Journal of Affective Disorders, 86(2), 205-213.

Kuyken, W., Warren, F. C., Taylor, R. S., Whalley, B., Crane, C., Bondolfi, G., et al. (2016). Efficacy of mindfulness-based cognitive therapy in prevention of depressive relapse: An individual patient data meta-analysis from randomized trials. JAMA Psychiatry. https://doi.org/10.1001/jamapsychiatry.2016.0076.

Lazarus, R. S. (1993). Coping theory and research: Past, present, and future. In Fifty years of the research and theory of RS Lazarus: An analysis of historical and perennial issues. UK: Psychology Press, pp 366-388.

LeMoult, J., Arditte, K. A., D’Avanzato, C., \& Joormann, J. (2013). State rumination: Associations with emotional stress reactivity and attention biases. Journal of Experimental Psychopathology, 4(5), 471.

Marchetti, I., Mor, N., Chiorri, C., \& Koster, E. H. (2018). The brief state rumination inventory (BSRI): Validation and psychometric evaluation. Cognitive Therapy and Research, 42, 1-14.

Michalak, J., Hölz, A., \& Teismann, T. (2011). Rumination as a predictor of relapse in mindfulness-based cognitive therapy for depression. Psychology and Psychotherapy: Theory, Research and Practice, 84(2), 230-236.

Michalak, J., Probst, T., Heidenreich, T., Bissantz, N., \& Schramm, E. (2016). Mindfulness-based cognitive therapy and a group version of the cognitive behavioral analysis system of psychotherapy for chronic depression: Follow-up data of a randomized controlled trial and the moderating role of childhood adversities. Psychotherapy and Psychosomatics, 85(6), 378-380.

Nolen-Hoeksema, S. (2000). The role of rumination in depressive disorders and mixed anxiety/depressive symptoms. Journal of Abnormal Psychology, 109(3), 504.

Nolen-Hoeksema, S., \& Morrow, J. (1991). A prospective study of depression and posttraumatic stress symptoms after a natural disaster: The 1989 Loma Prieta Earthquake. Journal of Personality and Social Psychology, 61(1), 115. 
Nolen-Hoeksema, S., Parker, L. E., \& Larson, J. (1994). Ruminative coping with depressed mood following loss. Journal of Personality and Social Psychology, 67(1), 92.

Nolen-Hoeksema, S., Wisco, B. E., \& Lyubomirsky, S. (2008). Rethinking rumination. Perspectives on Psychological Science, 3(5), 400-424.

Podsakoff, P. M., MacKenzie, S. B., Lee, J.-Y., \& Podsakoff, N. P. (2003). Common method biases in behavioral research: A critical review of the literature and recommended remedies. Journal of Applied Psychology, 88(5), 879.

Puterman, E., DeLongis, A., \& Pomaki, G. (2010). Protecting us from ourselves: Social support as a buffer of trait and state rumination. Journal of Social and Clinical Psychology, 29(7), 797-820.

Raes, F., \& Hermans, D. (2007). The revised version of the Dutch ruminative response scale. Unpublished Instrument.

Raes, F., Schoofs, H., Hoes, D., Hermans, D., Van Den Eede, F., \& Franck, E. (2009). 'Reflection'en'brooding'als subtypes van rumineren: Een herziening van de Ruminative Response Scale. Gedragstherapie, 42(3/4), 205-214.

Rush, A. J., Carmody, T., Ibrahim, H., Trivedi, M., Biggs, M., ShoresWilson, K., et al. (2006). Comparison of self-report and clinician ratings on two inventories of depressive symptomatology. Psychiatric Services, 57(6), 829-837.

Rush, A. J., Gullion, C. M., Basco, M. R., Jarrett, R. B., \& Trivedi, M. H. (1996). The inventory of depressive symptomatology (IDS): Psychometric properties. Psychological Medicine, 26(3), 477-486.

Schoofs, H., Hermans, D., \& Raes, F. (2010). Brooding and reflection as subtypes of rumination: Evidence from confirmatory factor analysis in nonclinical samples using the Dutch ruminative response scale. Journal of Psychopathology and Behavioral Assessment, 32(4), 609-617. https://doi.org/10.1007/s1086 2-010-9182-9.

Segal, Z. V., Williams, J., \& Teasdale, J. (2002). Mindfulness-based cognitive therapy for depression: A new approach to relapse prevention. New York: Guilford.

Segal, Z. V., Williams, J. M. G., \& Teasdale. (2012). Mindfulnessbased cognitive therapy for depression. Guilford: Guilford Press.

Shahar, B., Britton, W. B., Sbarra, D. A., Figueredo, A. J., \& Bootzin, R. R. (2010). Mechanisms of change in mindfulness-based cognitive therapy for depression: Preliminary evidence from a randomized controlled trial. International Journal of Cognitive Therapy, 3(4), 402-418.

Solhan, M. B., Trull, T. J., Jahng, S., \& Wood, P. K. (2009). Clinical assessment of affective instability: Comparing EMA indices, questionnaire reports, and retrospective recall. Psychological Assessment, 21(3), 425.

Southworth, F., Grafton, B., MacLeod, C., \& Watkins, E. (2017). Heightened ruminative disposition is associated with impaired attentional disengagement from negative relative to positive information: Support for the "impaired disengagement" hypothesis. Cognition and Emotion, 31(3), 422-434.

Strauss, C., Cavanagh, K., Oliver, A., \& Pettman, D. (2014). Mindfulness-based interventions for people diagnosed with a current episode of an anxiety or depressive disorder: A meta-analysis of randomised controlled trials. PLoS ONE. 9(4), e96110.

Torpey, D. C., \& Klein, D. N. (2008). Chronic depression: Update on classification and treatment. Current Psychiatry Reports, 10(6), $458-464$.

UK Network of Mindfulness-Based Teacher Trainers. (2015). Good practice guidance for teaching mindfulness-based courses. Retrieved from http://mindfulnessteachersuk.org.uk/pdf/teacherguidelines.pdf. Accessed 25 Jun 2018.

van Aalderen, J. R., Donders, A. R. T., Giommi, F., Spinhoven, P., Barendregt, H. P., \& Speckens, A. E. M. (2011). The efficacy of mindfulness-based cognitive therapy in recurrent depressed patients with and without a current depressive episode: A randomized controlled trial. Psychological Medicine, 42(05), 9891001. https://doi.org/10.1017/s0033291711002054.

van der Velden, A. M., Kuyken, W., Wattar, U., Crane, C., Pallesen, K. J., Dahlgaard, J., Fjorback, L. O., \& Piet, J. (2015). A systematic review of mechanisms of change in mindfulness-based cognitive therapy in the treatment of recurrent major depressive disorder. Clinical Psychology Review, 37, 26-39.

van Groenestijn, M., Akkerhuis, G., Kupka, R., Schneider, N., \& Nolen, W. (1999). Structured clinical interview for DSM-IV axis I disorders (SCID-I; Dutch version). Lisse: Swets \& Zeitlinger.

Van Vugt, M. K., Hitchcock, P., Shahar, B., \& Britton, W. (2012). The effects of mindfulness-based cognitive therapy on affective memory recall dynamics in depression: A mechanistic model of rumination. Frontiers in Human Neuroscience, 6, 257.

Veehof, M. M., Peter, M., Taal, E., Westerhof, G. J., \& Bohlmeijer, E. T. (2011). Psychometric properties of the Dutch Five Facet Mindfulness Questionnaire (FFMQ) in patients with fibromyalgia. Clinical Rheumatology, 30(8), 1045-1054.

Veenman, M. V. (2011). Alternative assessment of strategy use with self-report instruments: A discussion. Metacognition and Learning, 6(2), 205-211.

Verhage, F. (1964). Intelligence and age. Assen, The Netherlands: Van Gorcum.

Wiersma, J. E., van Oppen, P., Van Schaik, D., Van der Does, A., Beekman, A., \& Penninx, B. (2011). Psychological characteristics of chronic depression: A longitudinal cohort study. Journal of Clinical Psychiatry, 72(3), 288-294.

Wilson, T. D., \& Dunn, E. W. (2004). Self-knowledge: Its limits, value, and potential for improvement. Annual Review of Psychology. 55, 493-518.

Zoccola, P. M., \& Dickerson, S. S. (2012). Assessing the relationship between rumination and cortisol: A review. Journal of Psychosomatic Research, 73(1), 1-9.

Publisher's Note Springer Nature remains neutral with regard to jurisdictional claims in published maps and institutional affiliations. 